\title{
EVOLUTIONARY PROGRAMMING GUIDED BY ANALYTICALLY GENERATED SEEDS
}

\author{
Neil Crossley, Emanuel Kitzelmann, Martin Hofmann, Ute Schmid \\ Faculty Information Systems and Applied Computer Science, University of Bamberg, 96045 Bamberg, Germany \\ neil.crossley@stud.uni-bamberg.de, \{emanuel.kitzelmann,martin.hofmann,ute.schmid\}@uni-bamberg.de
}

Keywords: Recursive Functions; Analytical Inductive Programming; Evolutionary Programming.

\begin{abstract}
Evolutionary programming is the most powerful method for inducing recursive functional programs from input/output examples while taking into account efficiency and complexity constraints for the target program. However, synthesis time can be considerably high. A strategy which is complementary to the generate-and -test based approaches of evolutionary programming is inductive analytical programming where program construction is example-driven, that is, target programs are constructed as minimal generalization over the given input/output examples. Synthesis with analytical approaches is fast, but the scope of synthesizable programs is restricted. We propose to combine both approaches in such a way that the power of evolutionary programming is preserved and synthesis becomes more efficient. We use the analytical system IGOR2 to generate seeds in form of program skeletons to guide the evolutionary system ADATE when searching for target programs. In an evaluations with several examples we can show that using such seeds indeed can speed up evolutionary programming considerably.
\end{abstract}

\section{INTRODUCTION}

Inductive programming research is concerned with the synthesis of recursive programs from incomplete specifications. Target programs are typically declarative - mostly functional (Kitzelmann, 2009; Olsson, 1995), sometimes logical (Quinlan and CameronJones, 1995; Flener and Yilmaz, 1999). Specifications are typically given in the form of input/output examples for the desired behavior of the target program. In addition, constraints for code complexity or time efficiency can be provided. There are two distinct approaches to inductive programming: analytical and evolutionary inductive programming.

Analytical inductive programming is data-driven and often relies on specifications which consist only of a small set of positive input/output examples. A recursive program is learned by detecting recurrence relations in the input/output examples and generalization over these regularities (Summers, 1977; Kitzelmann and Schmid, 2006). Typically, analytical approaches are fast and they can guarantee certain characteristics for the constructed program such as mini- mality of the generalization with respect to the given examples and termination. However, the class of learnable programs is necessarily restricted to such problems which can be specified by small sets of input/output examples. The scope of learnable programs can be somewhat widened by allowing the use of background knowledge (Kitzelmann, 2009).

Evolutionary inductive programming is based on search through the hypothesis space of possible programs given some programming language. A hypothesis is returned as a solution if it performs sufficiently well on the input/output examples with respect to some measure of fitness, typically involving code length and time efficiency. In that sense, evolutionary programming is a specific variant of generate and test. The scope of programs learnable with an evolutionary approach is, in principle, unrestricted. But, generation times are typically high and there is no guarantee that the returned program is the optimal solution with respect to the fitness function.

Therefore, we propose to use analytical inductive programming to generate initial seeds for evolutionary programming. The combination of both ap- 
proaches should be such that if a solution can be generated by analytical means alone, this fast and reliable approach should be used exclusively. If the problem is out of scope for analytical programming, at least a partial solution could be provided which then can be used as input for program evolution. Based on some initial experiments (Crossley et al., 2009), we will present more extensive analyses in this paper.

In the following, we first describe the evolutionary programming system ADATE and the analytical programming system IGOR2 (Kitzelmann, 2009). Afterwards we will introduce different strategies for the analytical generation of program seeds with IGOR2 and their incorporation in ADATE. We will report results of our first experiments and give a short conclusion.

\section{ADATE}

ADATE (Olsson, 1995; Vattekar, 2006) was initially proposed in the nineties and has been continually extended. To our knowledge, it is the most powerful approach to inductive programming which is currently available. ADATE constructs programs in a subset of the functional language ML, called ADATE-ML. The problem specification presented to ADATE consists of: a set of data types and a set of primitive functions; a set of sample inputs; an evaluation function; an initial declaration of the goal function $f$. Sample inputs typically are input/output pairs. It is enough to give only positive examples, but it is additionally possible to provide negative examples. There are a number of predefined evaluation functions, each using different measures for syntactic complexity and time efficiency of the goal program. These are completed by a callback evaluation function given in the problem specification which evaluates the return value of an inferred function for a given input example. In general, the search heuristic is to prefer smaller and faster functions. As is typical for evolutionary approaches, there are sets of individuals which are developed over generations such that fitter individuals have more chances to reproduce. If no additional knowledge is provided, in contrast to usual approaches, ADATE starts with a single individual - the empty function $f$.

The function declarations of all constructed program candidates use the declaration of $f$, differing only in the program body. To construct program bodies, only the programming constructs available in ADATE-ML can be used together with additionally data types and primitive functions provided in the problem specification.

The search operators are transformations used in reproduction to generate new individuals. These transformations include: replacements of expressions in the program body, abstraction of expressions by introducing a call to a newly introduced function, distributing a function call currently outside a case expression over all cases, and altering the number and type of function arguments by various embedding techniques. From these ADATE constructs compound transformations, consisting of multiple atomic transformations, depending on the current stage of the search. Through management of an upper bound for the number of compound transformations used to generate individuals, ADATE can employ iterative deepening in its exploration of the problem space. In the current version of ADATE crossover is realized by applying a compound transformation from one individual to another (Vattekar, 2006).

In our experiments we used ADATE with the same set of only positive input/output examples which can be presented to the analytical system IGOR2. No data types and function primitives beyond ADATEML were used. Performance was evaluated with the predefined measures. Correctness was evaluated by a user-defined all-or-nothing function. The searchedfor program $f$ was presented either with an empty body or with an initial seed constructed by analytical induction. A simple example is given in figure 1.

With only the minimum necessary background knowledge, such as necessary data types and atomic functions, ADATE is able to find reasonable solutions given enough time. Additional background knowledge can reduce the required transformations to infer correct solutions, which can also reduce search time. However, additional background knowledge exacts deeper insights into the problem on behalf of the problem specifier. From a programming assistance system perspective, it would be not convenient to expect that a human programmer could provide exactly such knowledge which would increase the speed of generating a solution. This would presuppose (a) that the user understands the inner workings of ADATE and (b) has a deep insight in the programming problem at hand. From a cognitive perspective, such additional knowledge to guide ADATE's search might be gained by a closer inspection of the structure of the input/output examples, thereby providing ADATE with a helpful initial hypothesis.

\section{IGOR2}

IGOR2 (Kitzelmann, 2009) - to our knowledge - is currently the most powerful system for analytical inductive programming. Its scope of inducable programs and the time efficiency of the induction algo- 
Examples (represented in MAUDE notation):

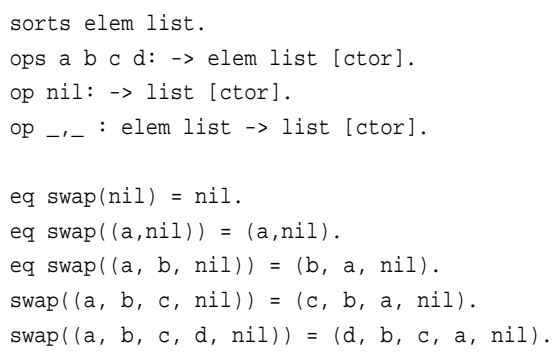

Program induced by IGOR2:

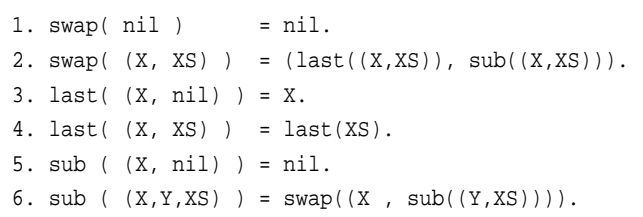

Best program induced by ADATE with empty seed:

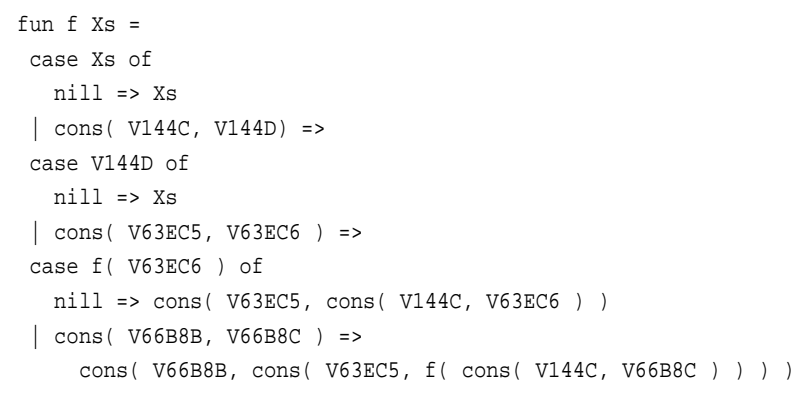

Figure 1: The Swap Function

rithm compares very well with classical approaches to inductive logic programming and other approaches to inductive programming (Hofmann et al., 2008). IGOR 2 continues the tradition of previous work in learning LISP functions from examples (Summers, 1977) as the successor to IGOR 1 (Kitzelmann and Schmid, 2006).

The system is realized in the constructor term rewriting system MAUDE. Therefore, all constructors specified for the data types used in the given examples are available for program construction. IGOR2 specifications consist of: a small set of positive input/output examples, presented as equations, which have to be the first examples with respect to the underlying data type and a specification of the input data type. Furthermore, background knowledge for additional functions can (but must not) be provided.

IGOR 2 can induce several dependent target functions (i.e., mutual recursion) in one run. Auxiliary functions are invented if needed. In general, a set of rules is constructed by generalization of the input data by introducing patterns and predicates to partition the given examples and synthesis of expressions comput- ing the specified outputs. Partitioning and searching for expressions is done systematically and completely which is tractable even for relatively complex examples because construction of hypotheses is datadriven. An example of a problem specification and a solution produced by IGOR 2 is given in figure 1 .

Considering hypotheses as equations and applying equational logic, the analytical method assures that only hypotheses entailing the provided example equations are generated. However, the intermediate hypotheses may be unfinished in that the rules contain unbound variables in the right-hand side (rhs), i.e., do not represent functions. The search stops, if one of the currently best hypotheses is finished, i.e., all variables in the rhss are bound.

IGOR2's built-in inductive bias is to prefer fewer case distinctions, most specific patterns and fewer recursive calls. Thus, the initial hypothesis is a single rule per target function which is the least general generalization of the example equations. If a rule contains unbound variables, successor hypotheses are computed using the following operations: (i) Partitioning of the inputs by replacing one pattern by a set of disjoint more specific patterns or by introducing a predicate to the righthand side of the rule; (ii) replacing the righthand side of a rule by a (recursive) call to a defined function (including the target function) where finding the argument of the function call is treated as a new induction problem, that is, an auxiliary function is invented; (iii) replacing subterms in the righthand side of a rule which contain unbound variables by a call to new subprograms.

Refining a Pattern. Computing a set of more specific patterns, case (i), in order to introduce a case distinction, is done as follows: A position in the pattern $p$ with a variable resulting from generalising the corresponding subterms in the subsumed example inputs is identified. This implies that at least two of the subsumed inputs have different constructor symbols at this position. Now all subsumed inputs are partitioned such that all of them with the same constructor at this position belong to the same subset. Together with the corresponding example outputs this yields a partition of the example equations whose inputs are subsumed by $p$. Now for each subset a new initial hypothesis is computed, leading to one set of successor rules. Since more than one position may be selected, different partitions may be induced, leading to a set of successor rule-sets.

For example, let

$$
\begin{array}{ll}
\text { reverse }([]) & =[] \\
\text { reverse }([X]) & =[X] \\
\text { reverse }([X, Y]) & =[Y, X]
\end{array}
$$

be some examples for the reverse-function. The pat- 
tern of the initial rule is simply a variable $Q$, since the example input terms have no common root symbol. Hence, the unique position at which the pattern contains a variable and the example inputs different constructors is the root position. The first example input consists of only the constant [] at the root position. All remaining example inputs have the list constructor cons as root. Put differently, two subsets are induced by the root position, one containing the first example, the other containing the two remaining examples. The least general generalizations of the example inputs of these two subsets are [] and $[Q \mid Q s]$ resp. which are the (more specific) patterns of the two successor rules.

Introducing (Recursive) Function Calls and Auxiliary Functions. In cases (ii) and (iii) help functions are invented. This includes the generation of I/O-examples from which they are induced. For case (ii) this is done as follows: Function calls are introduced by matching the currently considered outputs, i.e., those outputs whose inputs match the pattern of the currently considered rule, with the outputs of any defined function. If all current outputs match, then the rhs of the current unfinished rule can be set to a call of the matched defined function. The argument of the call must map the currently considered inputs to the inputs of the matched defined function. For case (iii), the example inputs of the new defined function also equal the currently considered inputs. The outputs are the corresponding subterms of the currently considered outputs.

For an example of case (iii) consider the last two reverse examples as they have been put into one subset in the previous section. The initial rule for these two examples is:

$$
\text { reverse }([Q \mid Q s])=[Q 2 \mid Q s 2]
$$

This rule is unfinished due to the two unbound variables in the rhs. Now the two unfinished subterms (consisting of exactly the two variables) are taken as new subproblems. This leads to two new examples sets for two new help functions sub1 and $s u b 2$ :

$$
\begin{aligned}
& \operatorname{sub1}([X])=X \quad \operatorname{sub2}([X])=[] \\
& \operatorname{sub1}([X, Y])=Y \quad \operatorname{sub2}([X, Y])=[X]
\end{aligned}
$$

The successor rule-set for the unfinished rule contains three rules determined as follows: The original unfinished rule (1) is replaced by the finished rule:

$$
\text { reverse }([Q \mid Q s])=[\operatorname{sub} 1([Q \mid Q s] \mid \operatorname{sub} 2[Q \mid Q s]]
$$

And from both new example sets an initial rule is derived.

Finally, as an example for case (ii), consider the example equations for the help function $s u b 2$ and the generated unfinished initial rule:

$$
\operatorname{sub2}([Q \mid Q s]=Q s 2
$$

The example outputs, []$,[X]$ of sub2 match the first two example outputs of the reverse-function. That is, the unfinished rhs $Q s 2$ can be replaced by a (recursive) call to the reverse-function. The argument of the call must map the inputs $[X],[X, Y]$ of $s u b 2$ to the corresponding inputs [], $[X]$ of reverse, i.e., a new help function, $s u b 3$ is needed. This leads to the new example set:

$$
\begin{array}{ll}
\operatorname{sub3}([X]) & =[] \\
\operatorname{sub3}([X, Y] & =[X]
\end{array}
$$

The successor rule-set for the unfinished rule contains two rules determined as follows: The original unfinished rule (2) is replaced by the finished rule:

$$
\operatorname{sub2}([Q \mid Q s]=\operatorname{reverse}(\operatorname{sub3}([Q \mid Q s]))
$$

Additionally it contains the initial rule for sub3.

\section{ANALYTICALLY GENERATED SEEDS FOR PROGRAM EVOLUTION}

As proposed above, we want to investigate whether using IGOR 2 as a preprocessor for ADATE can speedup ADATE's search for a useful program. Furthermore, it should be the case that the induced program should be as least as efficient as a solution found unassisted by ADATE with respect to ADATE's evaluation function. Obviously, coupling of IGOR2 with ADATE becomes only necessary in such cases where IGOR2 fails to generate a completed program. This occurs if IGOR 2 was presented with a too small set of examples or if analytically processing the given set of examples is not feasible within the given resources of memory and time. In these cases IGOR 2 terminates with an incomplete program which still contains unbound variables in the body of rules, namely, with missing recursive calls or auxiliary functions.

To have full control over our initial experiments, we only considered problems which IGOR 2 can solve fully automatically. We artificially created partial solutions by replacing function calls by unbound variables. We investigated the following strategies for providing ADATE with an initial seed:

For a given ADATE-ML program of the form

fun $f(\ldots):$ myType $=$ raise $D 1$

fun main $(\ldots)$ : mytype $=f(\ldots)$

- the function $f$ is redefined using the partial solution of IGOR2,

- or the problem space becomes restricted from the top-level by introducing the partial solution in the function main. 
- Any IGOR2 induced auxiliary functions can also be included: as an atomic, predefined function to be called by $f$ or as an inner function of $f$ also subject to transformations.

\section{EXPERIMENTS}

We presented examples of the following problems to IGOR2:

$\operatorname{addition}(\mathbf{X}, \mathbf{Y})=\mathbf{Z}$ iff $\mathbf{Z}$ is the sum of the two non-negative natural numbers $\mathrm{X}$ and $\mathrm{Y} . \mathrm{X}, \mathrm{Y}$ and $\mathrm{Z}$ are represented using peano numbers.

even_integer(X) is true iff $\mathrm{X}$ is an even number. $\mathrm{X}$ is a non-negative integer. even_peano(X) is true iff $\mathrm{X}$ is an even number. $\mathrm{X}$ is a non-negative peano number.

even_pos $(\mathbf{X})=\mathbf{Y}$ iff $\mathrm{Y}$ is a list containing all elements on even positions in list X.

$\operatorname{insert}(\mathbf{X}, \mathbf{Y})=\mathbf{Z}$ iff $\mathrm{X}$ is a list of elements sorted in an ascending order and $\mathrm{Z}$ is a list of elements $\mathrm{X}+\mathrm{Y}$ sorted in an ascending order.

$\operatorname{last}(\mathbf{X})=\mathbf{Y}$ iff $\mathrm{Y}$ is identical to list $\mathrm{X}$ without its last element.

lasts $(\mathbf{X})=\mathbf{Y}$ iff $\mathrm{X}$ is a list of lists and $\mathrm{Y}$ is a list containing the last element of each list in $\mathrm{X}$ in the order those lists appear in $\mathrm{X}$.

length_integer $(\mathbf{X})=\mathbf{Y}$ iff there are $\mathrm{Y}$ elements in the list $\mathrm{X}$. $\mathrm{Y}$ is an integer.

length_peano $(X)=Y$ iff there are $\mathrm{Y}$ elements in the list $\mathrm{X}$. $\mathrm{Y}$ is a peano number.

member $(\mathbf{X}, \mathbf{Y})$ is true iff $\mathrm{X}$ is also an element in the list $\mathrm{Y}$.

multlast $(X)=Y$ iff $Y$ is a list of equal length as the list $X$ and all its elements are identical to the last element in $\mathrm{X}$.

reverse $(\mathbf{X})=\mathbf{Y}$ iff $\mathrm{Y}$ is a list containing the same elements as $\mathrm{X}$, only in the reverse order.

$\operatorname{shiftL}(\mathbf{X})=\mathbf{Y}$ iff the list $\mathrm{Y}$ is identical to the list $\mathrm{X}$, except that the first element in $\mathrm{X}$ is on the last position in $\mathrm{Y}$ and all other elements are shifted one position to the left.

$\operatorname{shiftR}(\mathbf{X})=\mathbf{Y}$ iff the list $\mathrm{Y}$ is identical to the list $\mathrm{X}$, except that the last element in $\mathrm{X}$ is on the first position in $\mathrm{Y}$ and all other elements are shifted one position to the right.

$\operatorname{swap}(\mathbf{X})=\mathbf{Y}$ iff the list $\mathrm{Y}$ is identical to the list $\mathrm{X}$, except that the first and last element are swapped in around in $\mathrm{Y}$.

$\operatorname{swapalt}(\mathbf{X})=\mathbf{Y}$ iff the list $\mathrm{Y}$ is a permutation of the list $\mathrm{X}$, created by alternatingly removing the last and first element of $\mathrm{X}$ and adding them onto the end of the empty list.

$\operatorname{switch}(\mathbf{X})=\mathbf{Y}$ iff the list $\mathrm{Y}$ can be obtained from the list $\mathrm{X}$ by switching every second in $\mathrm{X}$ with the previous element.

switch2in3 $(X)=\mathbf{Y}$ iff the list $\mathrm{Y}$ can be obtained from the list $\mathrm{X}$ by switching the second element and every third thereafter with the previous element in X.

To generate an initial seed for ADATE, typically the righthand side of a recursive rule was replaced by an unbound variable. For example, the solution for switch provided by IGOR 2 was

$\operatorname{switch}([])=[]$

$\operatorname{switch}([\mathrm{X}])=[\mathrm{X}]$

$\operatorname{switch}([X, Y \mid X S])=[Y, X, \operatorname{switch}(X S)]$

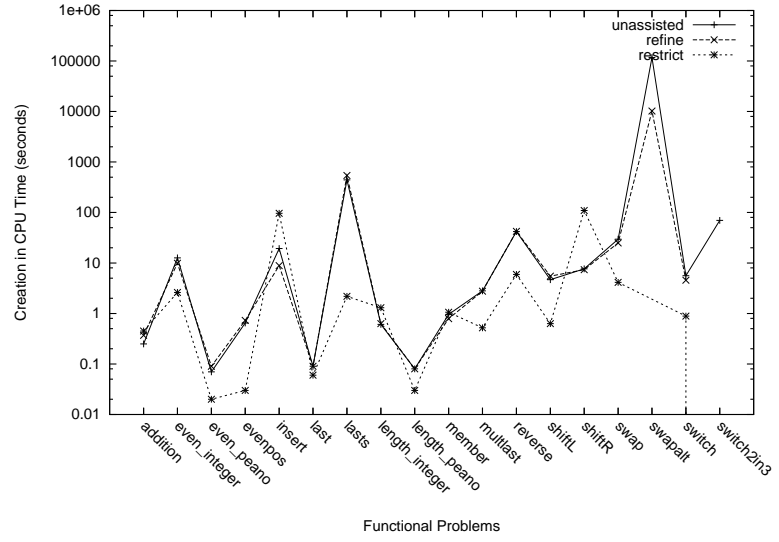

Figure 2: Creation times of best solutions

and the third rule was replaced by

$\operatorname{switch}([X, Y \mid X S])=Z$.

If IGOR2 induced solutions with auxiliary functions, either the function calls on the righthand side of the rules were made known to ADATE (see Sect. 4) or this information was obscured by again replacing the complete righthand side by a variable.

For example, for swap, IGOR2 inferred one atomic function last and inferred that the solution consists of two functions that recursively call each other as shown in figure 1. ADATE was presented with the rule 1, 2, 5 and 6 from figure 1 where the righthand side of rule 6 was replaced with an unbound variable.

Table 1: Overview of the solutions inferred by ADATE

\begin{tabular}{l|c|cc} 
& \# Rules & Auxiliars & \# Rules \\
\hline \hline \multicolumn{4}{c}{ neither better than unassisted } \\
\hline addition & 2 & 0 & 0 \\
insert & 3 & 0 & 0 \\
\hline \multicolumn{3}{c}{ only redefined better than unassisted } \\
\hline member & 3 & 0 & 0 \\
shiftR & 2 & 2 (init, last) & 4 \\
switch2in3 & 4 & 0 & 0 \\
\hline \multicolumn{4}{c}{ only restricted better than unassisted } \\
\hline even_peano & 3 & 0 & 0 \\
evenpos & 3 & 0 & 0 \\
lasts & 3 & 0 & 0 \\
length_peano & 2 & 0 & 0 \\
multlast & 2 & 1 (last) & 2 \\
reverse & 2 & 2 (last, revbutlast) & 4 \\
shiftL & 3 & 0 & 0 \\
\hline \multicolumn{2}{c}{ both redefined and restricted better than unassisted } \\
\hline even_integer & 3 & 0 & 0 \\
last & 2 & 0 & 0 \\
length_integer & 2 & 0 & 0 \\
swap & 2 & 2 (last, sub) & 4 \\
swapalt & 3 & 2 (ilast, take2nd) & 4 \\
switch & 3 & 0 & 0 \\
\hline
\end{tabular}


The results were ascertained by analysing the log files produced to document an ADATE run. ${ }^{1}$ To effectively compare the specifications we evaluated each according to the time taken to generate the most correct functions. Because ADATE infers many incorrect programs in the search process, we restricted our focus to those programs that:

- were tested by ADATE against the complete set of given training examples,

- terminated for each training example, and

- generated a correct output for each example.

This allowed us to achieve a meaningful overview of the performance of the specifications. For the analysis of the ADATE runs we considered the following information:

- the elapsed time since the start of the search until the creation of the program,

- the breakdown of the results the function produced for the examples, which in our case is the number of results evaluated as correct, incorrect or timed-out. Due to our evaluation restrictions, we filtered out all inferred functions which did not attain $100 \%$ correct results with the test examples.

- an ADATE time evaluation of the inferred function. This is the total execution time taken by the function for all the test examples as defined by ADATEs built in time complexity measure.

In most cases - with the exception of addition and insert - presenting ADATE with seeds generated by IGOR 2 resulted in faster inference times (see Figure 2).

A closer look at the results shows that quite a few problems benefited from both specification types - redefinition of the goal function $f$ with a program skeleton as well as restriction of ADATE's search space by introducing the skeleton into the main program (see Table 1). In most cases, it is clear that the right specification assistance improves the inference time for the best possible function. Gaining additional knowledge from the provided information, even using automatic analytical methods such as IGOR2, assists ADATE in producing acceptable results quicker than otherwise would be the case. Unfortunately, it is not clear which characteristic clearly categorises the problems into separate groups.

\footnotetext{
${ }^{1}$ Technical details are given in a report by $\mathrm{N}$. Crossley available at http://www.cogsys.wiai.uni-bamberg.de/ teaching/ss07/p_cogsys/adate-report.pdf.
}

\section{CONCLUSIONS}

We presented experiments where we can show that providing evolutionary programming with analytically constructed seeds constrains program synthesis such that search time can considerably reduced. We compared two possibilities for providing ADATE with program skeletons constructed with our analytical system IGOR2 and we could show that both approaches can improve performance. However, up to now we have not found a unique criterion to decide when to prefer which possibility. In future work we plan to combine ADATE and IGOR2 in such a way that IGOR 2 automatically can call ADATE to help for problems where it cannot find a recursive program by analytical means.

\section{REFERENCES}

Crossley, N., Kitzelmann, E., Hofmann, M., and Schmid, U. (2009). Combining analytical and evolutionary inductive programming. In Goerzel, B. et al., editors, Proc. of the 2nd Conference on Artificial General Intelligence (AGI-09), pages 19-24, Amsterdam. Atlantis.

Flener, P. and Yilmaz, S. (1999). Inductive synthesis of recursive logic programs: Achievements and prospects. Journal of Logic Programming, 41(2-3):141-195.

Hofmann, M., Kitzelmann, E., and Schmid, U. (2008). Analysis and evaluation of inductive programming systems in a higher-order framework. In Dengel, A., et al., editors, KI 2008: Advances in Artificial Intelligence, number 5243 in LNAI, pages 78-86, Berlin. Springer.

Kitzelmann, E. (2009). Analytical inductive functional programming. In Hanus, M., editor, Proc. of the 18th International Symposium on Logic-Based Program Synthesis and Transformation (LOPSTR 2008), volume 5438 of $L N C S$, pages 87-102. Springer.

Kitzelmann, E. and Schmid, U. (2006). Inductive synthesis of functional programs: An explanation based generalization approach. Journal of Machine Learning Research, 7(Feb):429-454.

Olsson, R. (1995). Inductive functional programming using incremental program transformation. Artificial Intelligence, 74(1):55-83.

Quinlan, J. and Cameron-Jones, R. (1995). Induction of logic programs: FOIL and related systems. New Generation Computing, Special Issue on Inductive Logic Programming, 13(3-4):287-312.

Summers, P. D. (1977). A methodology for LISP program construction from examples. Journal ACM, 24(1):162-175.

Vattekar, G. (2006). Adate User Manual. Technical report, Ostfold University College. 